This is an author produced version of a paper published in Fertility and sterility. This paper has been peer-reviewed but does not include the final publisher proofcorrections or journal pagination.

Citation for the published paper:

Axmon, Anna and Hagmar, Lars

"Time to pregnancy and pregnancy outcome."

Fertil Steril. 2005 Oct;84(4):966-74.

http://dx.doi.org/10.1016/j.fertnstert.2005.04.030

Access to the published version may require journal subscription.

Published with permission from: Elsevier 


\title{
Time to pregnancy and pregnancy outcome
}

\author{
Anna Axmon, PhD, Lars Hagmar, MD
}

Division of Occupational and Environmental Medicine and Psychiatric Epidemiology, University Hospital, SE-221 85 Lund, Sweden

This work was financed by grants from the Swedish Research Council, the Swedish Research Council for Environment, Agricultural Sciences and Spatial Planning, the Swedish Council for Working Life and Social Research, the Medical Faculty at Lund University, and Region Skåne.

Reprint requests to: Anna Axmon, Division of Occupational and Environmental Medicine and Psychiatric Epidemiology, University Hospital, SE-221 85 Lund, Sweden. Tel +46-46-173960, fax+46-46-173669, e-mail anna.axmon@med.lu.se

\begin{abstract}
OBJECTIVE: To investigate whether the outcome of a pregnancy is related to the time required to achieve that pregnancy (TTP).

DESIGN: The distribution of the TTP for pregnancies ending in multiple birth, early (before week 12) and late (weeks 12-28) miscarriage, stillbirth, and extrauterine pregnancy was compared to that of pregnancies ending in singleton birth. Furthermore, the distribution of the TTP for preterm singleton births was compared to that of fullterm singleton births.
\end{abstract}

SETTING: Sweden

PATIENTS: Information from three previous studies on reproduction was used: Women chosen due to exposure to persistent organochlorine pollutants, or exposure as a hairdresser, and their respective controls.

INTERVENTION(S): None

MAIN OUTCOME MEASURES: Self-reported pregnancy outcome.

RESULTS: An increased TTP (i.e. decreased fecundability) was associated with pregnancies ending in miscarriage (early as well as late) and extrauterine pregnancies. Pregnancies ending in multiple live birth tended to have shorter TTPs than those ending in single live birth. No association between TTP and stillbirths was found. Among women whose pregnancies ended in singleton birth, a prolonged TTP was associated with preterm delivery.

CONCLUSIONS: The TTP of a pregnancy seemed to be associated with the outcome of that pregnancy. The mechanisms behind this phenomenon are, however, unclear.

KEY WORDS (MeSH): Fertility / Pregnancy Outcome / Gestational Age 


\section{INTRODUCTION}

The time needed to achieve a wanted pregnancy - time to pregnancy (TTP) - can be used to estimate fecundability (1), and has been found to be a useful tool for the assessment of reproductive effects (2). It is easily achievable information, which can be collected retrospectively (2) by using self-administered questionnaires (3). However, as easy as TTP is to inquire about, as difficult is it to explain the complex mechanisms of biological, sociological and environmental factors that may affect TTP. However, by investigating the possible association between TTP and pregnancy outcome, the understanding of these mechanisms may be facilitated (4).

Previous studies have found that pregnancies ending in miscarriage took longer to achieve than those ending in live birth $(4,5)$, and that miscarriages were more common among subfertile women (6). With respect to twin births, a recent study has found a reduced risk associated with a long TTP (7). Furthermore, pregnancies with a long TTP (4) and pregnancies among subfertile women (8, 9) were more likely to end with preterm delivery than to be full term.

The aim of the present study was to further explore a possible association between TTP and the risk of preterm delivery, as well as different pregnancy outcomes such as miscarriage, stillbirth, multiple birth, and extrauterine pregnancies.

\section{MATERIALS AND METHODS}

\section{Study population}

The present study is based on information collected in three previous studies, which were all approved by the Ethics Committee at Lund University. In total, 5302 women were included in the present study.

\section{Fishermen's wives}

In 1997, a self-administered questionnaire which included questions on TTP and pregnancy outcome was sent to women, born in 1945 or later, who were married to fishermen from the Swedish east coast. The rationale for the use of this group of women was that the fish caught off the Swedish east coast (i.e. the Baltic Sea) is contaminated by persistent organochlorine compounds (POCs). Indeed, this fish an important source of exposure to POCs in the Swedish population (10-12). Previous studies had found that Swedish fishermen and their families on average consume more fish than the general Swedish population $(10,13,14)$. Thus, the east coast fishermen's wives were considered an appropriate study group to investigate health effects from dietary POC exposure.

Fishermen's wives from the Swedish west coast have been found to be similar to the east coast wives with respect to socioeconomic factors, as well as fish consumption (10, 13-15). However, the fish caught on the west coast is less contaminated (15). Therefore, the west coast wives were considered as a appropriate referent cohort. Note that we have refrained from using the term "non-exposed". Since POCs are so widely distributed in the environment there is virtually no person who is non-exposed to these compounds. The number of respondents to the questionnaire was 505 east coast and 1090 west coast women (Table 1).

The information collected through the questionnaires was used to investigate the relation between on one hand POC exposure and on the other TTP (16), miscarriages (17), and menstrual cycle length (18).

\section{Fishermen's sisters}

Similar to the fishermen's wives, sisters to Swedish fishermen have also a high average fish consumption $(10,13,14)$. Fur- 
thermore, they are more likely to have grown up in a fisherman's family and/or fishing village than fishermen's wives and women from the general Swedish population (19). Thus, east coast fishermen's sisters are likely to have been exposed to POCs during their childhood and adolescence. Again, the corresponding group of women from the Swedish west coast was regarded as a suitable referent cohort.

In 1999, self-administered questionnaires which included questions on TTP and pregnancy outcome were sent to fishermen's sisters born between 1945 and 1979. The number of respondents were 709 and 1103 in the east and west coast cohort, respectively (Table 1). As with the fishermen's wives the objective of the study was to investigate the relation between POC exposure and the outcomes TTP, miscarriage (20), and menstrual cycle length (18).

\section{Hairdressers/general population}

In order to assess health effects of working as a hairdresser, a cohort of women who had graduated from Swedish vocational schools for hairdresser was established (21). As referents, age-matched women from the general Swedish population were chosen. In 2000 , all women born in 1960, or later, in the two cohorts were sent self-administered questionnaires including questions on TTP and pregnancy outcome. The number of respondents in the hairdresser cohort was 2626 and in the general population cohort 2860 (Table 1).

The information collected through the questionnaires will be used to study reproductive outcomes among hairdressers (Axmon et al., unpublished data, 2005).

\section{Time to pregnancy}

Information on TTP was collected somewhat differently in the three studies. Whereas the fishermen's wives were simply asked "How many months did it take you to become pregnant?", a line of questions were used to establish TTP among the fishermen's sisters and hairdressers/general population: "Did you become pregnant the first month of trying? If no, did you become pregnant the second month of trying? If no, in which month did you become pregnant?”.

All women were asked the question "Was the pregnancy a result of any medical treatment?". The women who answered "yes" to this question $(n=263)$ were excluded from all analyses since infertility treatment in itself can be a risk factor for adverse pregnancy outcome.

Of the women who responded to the questionnaire, the fraction of women who provided a valid TTP varied between 52\% and $86 \%$ (total $66 \%$; c.f. Table 1).

\section{Pregnancy outcome and preterm delivery}

For the fishermen's sisters and hairdressers/general population, pregnancy outcome was assessed as one live born child, more than one live born child, induced abortion, extrauterine pregnancy, miscarriage, stillborn child, or still pregnant. Furthermore, information was collected on the gestational length of the pregnancy. In the fishermen's wives questionnaire, live births were categorized as boy, girl, identical twins, or non-identical twins. There was no question about gestational length, but for pregnancies that ended in a miscarriage it was established if this was before week 12 , weeks $12-28$ or after week 28.

For the purpose of this study, the outcome was categorized as pregnancies ending with singleton live birth, multiple live birth, early miscarriage (before week 12), late 
miscarriage (weeks 12-28), stillbirth (after week 28), or extrauterine pregnancy. Note that for multiple births there were no information on the number of infants born. Sixtyeight women had provided a TTP for a pregnancy that ended in induced abortion. Since induced abortions are most common in unwanted pregnancies, i.e. pregnancies for which a TTP is unavailable, these 68 pregnancies were excluded from the analyses, as was the 154 still ongoing pregnancies.

In the questionnaires sent to the fishermen's sisters and hairdressers/general population, information was collected on the gestational week in which the pregnancy ended. It was not stated in the questionnaire if the length of the pregnancy was determined by last menstrual period or by ultrasound examination. Singleton births before gestational week 37 were considered as preterm deliveries.

\section{Possible confounders and effect modifiers}

Information on the year of the pregnancy, the woman's age at conception, use of oral contraceptives before trying to get pregnant, parity of the first planned pregnancy (recorded as first or higher), and smoking habits prior to the pregnancy $(0,1-$ $9,10-19$, or $20+$ cigarettes per day) were collected in all three questionnaires (Table 2).

The hairdressers/general population had their pregnancies at a later date than both the fishermen's wives and fishermen's sisters. Furthermore, the hairdressers/general population were slightly older in their first planned pregnancy than the two other groups of women. The three groups differed in smoking habits, with the fraction of nonsmokers ranging between 48 and $76 \%$. The fraction of oral contraceptive users also differed between the groups. However, the number of nulliparous women was similar.
All variables presented in Table 2 were considered as potential confounders.

The original study from which the information was collected, and cohort affiliation in the original study (exposed/referents), were considered as potential confounders as well as effect modifiers.

\section{Non-respondents}

The response rates in the three studies varied between 50\% and 64\% (Table 1). By linking the Swedish Medical Birth Registry to the fishermen's wives cohorts, respondents and non-respondents were compared with respect to reproductive outcomes such as ever having given birth, ever born a lowbirth-weight or malformed infant, as well as smoking habits and age $(16,17)$. It was found that the distributions of these factors were similar among the respondents and the non-respondents. However, using the same technique in the fishermen's sisters study revealed that the non-respondents tended to be older, less educated, and less likely to ever having given birth than the responders (20).

In the hairdresser study the respondents and non-respondents did not differ in age, or ever having given birth (Axmon et al., unpublished data, 2005). However, there was a lower fraction of nonsmokers among the non-respondents than among the respondents.

\section{Statistics}

Logistic regression was employed to estimate the association between TTP and pregnancy outcome and preterm delivery. For each comparison, a fecundability ratio (FR) was calculated from the odds ratio (OR) for pregnancy of each time interval (11.9, 2-2.9 months, etc). The FR can be used to estimate differences in TTP between two 
groups, i.e. FR 0.87 indicates a $15 \%$ longer TTP $(1 / 0.87=1.15)$. The TTP distribution of the different pregnancy outcomes were compared to that of pregnancies ending in singleton live birth. Similarly, the TTP distribution of preterm singleton births was compared to that of fullterm singleton births.

Each woman was allowed to contribute with only one pregnancy to avoid interference from correlation between subsequent pregnancies. In order to increase the number of pregnancies studied, the first planned pregnancy was chosen, regardless of the parity of that pregnancy.

In the analyses, TTP was censored at 12 months. Some of the fishermen's sisters and hairdressers/general population had stated that they did not become pregnant neither the first nor the second month of trying, but had not provided a TTP. These women were included in the analyses, but censored at 2 months (fishermen's sisters, $n=46$; hairdressers/general population, $\mathrm{n}=52$ ).

Women whose pregnancies are unplanned may change their feelings about the pregnancy once it is recognized. These women tend to report their TTP as one month (22). To control for this "birth control bias", analyses were performed excluding women who stated that they became pregnant the first month of trying.

Although women, on a group level, have been found to remember their TTP with surprising accuracy (23), some of the women included in the present study have been asked to remember TTPs stretching as far as 30 years back. Therefore, subgroup analyses were performed including only women whose first planned pregnancy was as most ten $(n=2368)$ or five $(n=1090)$ years before answering the questionnaire.

Multivariate analyses were performed including the potential confounders described above. When selecting confounders for the multivariate analyses, the method suggested by Greenland (24) was used. Starting with the univariate model, variables were entered into bivariate and multivariate models if they changed the effect estimate by $10 \%$ or more, and excluded if exclusion changed the effect estimate by less than $5 \%$.

Stratified analyses were performed for the potential effect modifiers.

\section{RESULTS}

\section{Miscarriages and stillbirths}

Compared to pregnancies ending in singleton birth, pregnancies ending in miscarriage (early as well as late) took longer to achieve (Table 3; Figure 1). The results were similar when excluding first month conceptions. Among women with at most ten years recall, 130 had experienced an early and 97 a late miscarriage. The corresponding numbers for women with at most 5 years recall were 69 and 51 . The results from these subgroups of women were similar to those from the entire group of women, i.e. when recall time was not taken into consideration.

There were no apparent differences in TTP between pregnancies ending in single live birth and those ending in stillbirth. None of the analyses were affected by the introduction of the potential confounders, neither did stratifying on original study or original cohort affiliation change the patterns of association (Table 4).

\section{Extrauterine pregnancies}

The TTP for extrauterine pregnancies was increased compared to that of pregnancies ending in live birth (FR 0.45; 0.280.71; Table 3; Figure 2). The results were still statistically significant when excluding first month conceptions (FR 0.57; 0.340.95). Furthermore, the results for the 15 
women with recall time at most ten years, and the eight women with recall time at most five years, the results were similar to those found in the entire group of women. None of the potential confounders changed the estimate more than $10 \%$. Also, the results were similar when the material was stratified on the original study and cohort affiliation in original study (Table 4).

\section{Multiple birth}

Pregnancies ending in multiple birth had shorter TTPs than pregnancies ending in singleton birth (FR 1.62; 1.51-2.27; Table 3; Figure 2). However, the effect was not as obvious when excluding first month conceptions (FR 1.10; 0.64-1.89). When analyzing the subgroups of women with at most ten $(n=29)$ or five $(n=13)$ years recall, the results were similar as when the recall time was not taken into consideration. The effect estimates were not changed by the introduction of potential confounders. Furthermore, the results were similar when the material was stratified on the original study and cohort affiliation in original study (Table 4).

\section{Preterm delivery}

Among the fishermen's sisters and hairdressers/general population, 255 women (7\%) with pregnancies ending in singleton birth gave birth prior to gestational week 37 . Giving birth preterm was associated with a prolonged TTP (FR 0.86; CI 0.73-1.01). The results were similar for hairdressers/general population (FR 0.89; 0.72-1.10) and fishermen's sisters (FR 0.81; 0.63-1.04). Furthermore, when stratifying on cohort affiliation in the original study, the results were similar for referents (FR 0.83; 0.66-1.03) and exposed (FR 0.89; 0.71-1.13).

Similar results were also found when excluding first month conceptions (FR 0.83; $0.68-1.01$ ), and when restricting the analyses to those women who had at most ten $(\mathrm{n}=122$; FR 0.87; 0.69-1.09) or five $(n=62$; FR 0.79; $0.58-1.09$ ) years of recall time. None of the potential confounders changed the effect estimates more than marginally.

\section{DISCUSSION}

A prolonged TTP was found for pregnancies ending in miscarriage (early as well as late) compared to pregnancies ending with the birth of a live child. Prolonged TTPs were also found for extrauterine pregnancies, whereas TTP for pregnancies ending in multiple birth was shorter than that for pregnancies ending in singleton live birth. Among women with pregnancies ending in singleton birth, a long TTP was a risk factor for preterm delivery.

TTP has been found to be a good estimate of fecundability (1) and to be a useful tool when assessing effects on reproduction (25, 26). Information concerning TTP can be collected retrospectively (2) using self-administered questionnaires (3), and women have, on a group level, been found to remember their TTP with surprising accuracy (23). In the present study, some women have been asked about pregnancies that occurred up to 30 years ago. This is a long time to remember circumstances surrounding a pregnancy, especially if the pregnancy did not end with a live birth. However, subgroup analyses were performed using only women whose recall time was at most ten or five years, respectively. The fecundability estimates achieved from these analyses were similar to those found when analyzing all women regardless of their recall time.

The way in which TTP was established among the fishermen's wives allowed for the women to state that they became pregnant after zero months of trying. However, it has been found that this does not have any major impact on the effect estimates for TTP (27). 
Thus, performing joint analyses of the three cohorts should not pose a problem with respect to this. However, the cohorts of fishermen's wives and fishermen's sisters probably does not fully represent women from the general Swedish population, but are rather to be considered as a homogenous group with respect to both socio-economic and dietary factors. On the other hand, the hairdressers and their referents may very well be considered a representative sample of the general Swedish female population. When stratifying on original study, the results from the hairdresser/referent cohort were very similar to those found when analyzing the entire group of women, irrespective of original study. Thus, our findings seem to be of a general nature and not restricted to any specific subgroup of women, or associated with any specific exposure, and performing joint analyses should not be a problem with this respect either.

Studies of TTP normally exclude women whose pregnancies are unplanned, since no proper TTP can be given for these pregnancies. Since the data in the present study was collected in three studies investigating the effect of different exposures on TTP, the focus was obviously on planned pregnancies. When assessing a possible relation between TTP and pregnancy outcome, it may be of interest to include also unplanned pregnancies. By excluding these pregnancies, we are excluding the most fertile women. The present study strongly indicates that the pregnancies of highly fertile women (i.e. women with short TTPs) are more likely to end with multiple birth, whereas pregnancies of less fertile women are more likely to end with a miscarriage or an extrauterine pregnancy. Thus, including women with unplanned pregnancies would probably strengthen our results.

There were a few differences among respondents and non-respondents in the pre- sent study. In two of the three studies from which data was collected, the nonrespondents were less likely to ever having given birth than the respondents (Axmon et al., unpublished data, 2005)(20). One way to interpret this is that women with decreased fertility were disinclined to participate in the studies. If decreased fertility means a prolonged TTP as well as an increased miscarriage risk, the results from the present study would underestimate the association between TTP and miscarriage risk.

Of the women who responded to the questionnaire, the fraction of women who provided a valid TTP varied between 52\% and $86 \%$ (total 66\%; c.f. Table 1). The lowest fraction was among the hairdressers (61\%) and their referents (52\%). This can be explained by the fact that these women were younger than the others. In fact, $33 \%$ of the hairdressers and $42 \%$ of the women from their referents claimed never to have tried to become pregnant (this information was not available for the fishermen's wives or the fishermen's sisters).

We performed subgroup analyses excluding first month conceptions. The rationale for this is to reduce birth control bias, i.e. that one group of women is more likely to claim that pregnancies due to birth control failure in fact were planned pregnancies (22). In the present study, we felt that these subgroup analyses were extra important, since the outcome of a pregnancy is highly likely to influence the woman's feelings about that pregnancy. For miscarriages, multiple births and extrauterine pregnancies, the results changed only marginally when excluding first month conceptions. For stillbirths, the shorter TTP associated with this outcome was prolonged when excluding first month conceptions. However, the confidence intervals were very wide for both fecundability estimates, and the shift from short to long TTP may very well be a random finding. 
Pregnancies ending in miscarriage or stillbirth took on average 12-35\% longer to achieve than those resulting in the birth of a live child (i.e. FR 0.75-0.89 in Table 3). These results are similar to the $23 \%$ increase in TTP for pregnancies ending in miscarriage found by Joffe and $\mathrm{Li}$ (4), but somewhat shorter than the 68\% longer median TTP found among Danish workers (5). The present results are also supported by a study among women from the USA, where the odds ratio for miscarriage among subfertile women compared to women without impaired fertility was 1.71 (6).

From a biological point of view, it is not surprising that an association with TTP should be found for pregnancies ending in early miscarriage. TTP is a measure that incorporates several functions in the woman, man and fetus, one of these being early survival of the fetus after fertilization. The line between a prolonged TTP and an early miscarriage is thus in part drawn considering the woman's ability to detect pregnancy. Furthermore, it has been suggested that the causation of embryonic and early fetal loss may share common underlying mechanisms (4).

Extrauterine pregnancies took more than twice the time to achieve as pregnancies ending in a live birth. Although these pregnancies were four times more likely to have occurred after infertility treatment, the results remained unchanged when excluding such pregnancies. Progesterone plays an essential role in the transport of the embryo to the uterus (28), and women with extrauterine pregnancies has been found to have lower progesterone levels than those with normal intrauterine pregnancies (29). Studies have found that lower midluteal progesterone is associated with failure to conceive (30, 31). Thus, low progesterone levels may be a shared risk factor for delayed conception and extrauterine pregnancies.
We found a $62 \%$ shorter average TTP for pregnancies ending with multiple live birth compared to those ending with singleton live birth. To the best of our knowledge, there are no previous studies that have assessed the relation between TTP as a continuous variable and multiple birth as an outcome. However, a recent Danish study compared women with long TTPs (3-5 months, 6-12 months, and >12 months) to women with short TTPs (0-2 months) and found decreasing risks for twin births with longer TTPs (7).

Singleton pregnancies that ended in preterm delivery took on average $16 \%$ longer to achieve than fullterm singleton pregnancies. This is in agreement with Joffe and $\mathrm{Li}$ who found a $15 \%$ longer TTP among women with preterm labor (4). Our results are also supported by two Danish studies, which found increased risks (odds ratios between 1.3 and 1.9) for preterm delivery among subfertile women $(8,9)$.

We categorized singleton deliveries as preterm or fullterm based on the woman's own statement regarding the gestational length. It is unknown to us if these statements on gestational length were based on last menstrual period or ultrasonic examination, and also if there was a time trend with respect to which method was used (i.e. if the length of later pregnancies were more likely to have been determined by ultrasonic examination). Whenever last menstrual period is used to determine the gestational length, errors may be introduced by late recognition of the pregnancy, e.g. due to a bleeding in early pregnancy. A late recognition of the pregnancy would result in an overestimated TTP and an underestimated gestational length, which in turn would give a false association between TTP and preterm delivery.

In the present data, the prevalence of preterm births is 6-7\% between 1980 and 
1999, nearly 15\% in 1975 through 1980 and 25-30\% between 1965 and 1975. Thus, there is either a time trend with respect to preterm births, or misclassification of data. If the latter is true, the misclassification is most likely to be for earlier pregnancies, since the prevalence of preterm birth in recent years is in agreement with previous findings of 6$10 \%$ (32). Restricting the analyses to women whose recall time was at most five years (i.e. pregnancies no earlier than 1994) gave similar results as the analyses of the entire group of women, regardless of recall time. Hence, the possible misclassification of preterm birth is not dependent on the TTP.

In summary, extrauterine pregnancies and pregnancies ending in miscarriage seem to take longer to achieve than pregnancies ending in a live birth, whereas pregnancies ending in multiple birth took a shorter time to achieve than those ending in the birth of one live child. Furthermore, pregnancies that took a long time to achieve were more likely to end in preterm birth.

Previous studies on TTP and pregnancy outcome are restricted to comparisons between pregnancies ending in singleton live birth and pregnancies ending in miscarriage (4-6) or with a twin birth (7). Furthermore, studies investigating a possible association between TTP and preterm delivery are few $(4,8,9)$. The results from the present study do, nevertheless, support previous findings. Studies comparing the TTP for pregnancies ending in live singleton birth to that of extrauterine pregnancies and pregnancies ending in stillbirth have, to the best of our knowledge, never previously been performed.

\section{REFERENCES}

1. Weinberg CR, Gladen BC. The betageometric distribution applied to comparative fecundability studies. Biometrics 1986;42:547-60.
2. Joffe M. Feasibility of studying subfertility using retrospective self reports. J Epidemiol Community Health 1989;43:268-74.

3. Zielhuis GA, Hulscher ME, Florack EI. Validity and reliability of a questionnaire on fecundability. Int J Epidemiol 1992;21:11516.

4. Joffe M, Li Z. Association of time to pregnancy and the outcome of pregnancy. Fertil Steril 1994;62:71-5.

5. Schaumburg I, Boldsen JL. Waiting time to pregnancy and pregnancy outcome among Danish workers in the textile, clothing, and footwear industries. Scand J Soc Med 1992;20:110-4.

6. Gray RH, Wu LY. Subfertility and risk of spontaneous abortion. Am J Public Health 2000;90:1452-4.

7. Basso O, Christensen K, Olsen J. Fecundity and twinning. A study within the Danish National Birth Cohort. Hum Reprod 2004;19:2222-6.

8. Henriksen TB, Baird DD, Olsen J, Hedegaard M, Secher NJ, Wilcox AJ. Time to pregnancy and preterm delivery. Obstet Gynecol 1997;89:594-9.

9. Basso O, Baird DD. Infertility and preterm delivery, birthweight, and Caesarean section: a study within the Danish National Birth Cohort. Hum Reprod 2003;18:2478-84.

10. Svensson BG, Nilsson A, Hansson M, Rappe C, Akesson B, Skerfving S. Exposure to dioxins and dibenzofurans through the consumption of fish. $\mathrm{N}$ Engl $\mathrm{J}$ Med 1991;324:8-12.

11. Ahlborg UG, Lipworth L, Titus-Ernstoff L, et al. Organochlorine compounds in relation to breast cancer, endometrial cancer, and endometriosis: an assessment of the biological and epidemiological evidence. Crit Rev Toxicol 1995;25:463-531.

12. Asplund L, Svensson BG, Nilsson A, et al. Polychlorinated biphenyls, 1,1,1-trichloro2,2-bis(p-chlorophenyl)ethane (p,p'-DDT) and 1,1-dichloro-2,2-bis(p-chlorophenyl)ethylene (p,p'-DDE) in human plasma related to fish consumption. Arch Environ Health 1994;49:477-86.

13. Rylander L, Stromberg U, Hagmar L. Decreased birthweight among infants born to women with a high dietary intake of fish contaminated with persistent organochlorine compounds. Scand J Work Environ Health 1995;21:368-75.

14. Hagmar L, Linden K, Nilsson A, et al. Cancer incidence and mortality among 
Swedish Baltic Sea fishermen. Scand J Work Environ Health 1992;18:217-24.

15. Bergqvist P, Bergek S, Hallbäck H, Rappe C, Slorach S. Dioxins in cod and herring from the seas around Sweden. Chemosphere 1989:513-516.

16. Axmon A, Rylander L, Strömberg U, Hagmar L. Time to pregnancy and infertility among women with a high intake of fish contaminated with persistent organochlorine compounds. Scand J Work Environ Health 2000;26:199-206.

17. Axmon A, Rylander L, Strömberg U, Hagmar L. Miscarriages and stillbirths in women with a high intake of fish contaminated with persistent organochlorine compounds. Int Arch Occup Environ Health 2000;73:204-8.

18. Axmon A, Rylander L, Stromberg U, Hagmar L. Altered menstrual cycles in women with a high dietary intake of persistent organochlorine compounds. Chemosphere 2004;56:813-9.

19. Rylander L, Stromberg U, Hagmar L. Lowered birth weight among infants born to women with a high intake of fish contaminated with persistent organochlorine compounds. Chemosphere 2000;40:1255-62.

20. Axmon A, Rylander L, Strömberg U, Hagmar L. Female fertility in relation to the consumption of fish contaminated with persistent organochlorine compounds. Scand J Work Environ Health 2002;28:124-32.

21. Albin M, Rylander L, Mikoczy Z, et al. Incidence of asthma in female Swedish hairdressers. Occup Environ Med 2002;59:119-23.

22. Weinberg CR, Baird DD, Wilcox AJ. Sources of bias in studies of time to pregnancy. Stat Med 1994;13:671-81.

23. Joffe M, Villard L, Li Z, Plowman R, Vessey M. Long-term recall of time-topregnancy. Fertil Steril 1993;60:99-104.

24. Greenland S. Modeling and variable selection in epidemiologic analysis. Am J Public Health 1989;79:340-9.

25. Baird DD, Wilcox AJ, Weinberg CR. Use of time to pregnancy to study environmental exposures. Am J Epidemiol 1986;124:47080.

26. Joffe M. Time to pregnancy: a measure of reproductive function in either sex. Asclepios Project. Occup Environ Med 1997;54:289-95.

27. Axmon A. Fertility and female dietary exposure to persistent organochlorine compounds. Dept. of Occupational and Environmental Medicine, Inst. of Laboratory Medicine. Lund: Lund University, 2003:105.

28. De Cecco L, Capitanio GL, Croce S, Forcucci M, Gerbaldo D, Rissone R. Biology of nidation and ectopic implantation. Acta Eur Fertil 1984;15:347-55.

29. Dumps P, Meisser A, Pons D, et al. Accuracy of single measurements of pregnancy-associated plasma protein-A, human chorionic gonadotropin and progesterone in the diagnosis of early pregnancy failure. Eur J Obstet Gynecol Reprod Biol 2002;100:174-80.

30. Stewart DR, Overstreet JW, Nakajima ST, Lasley BL. Enhanced ovarian steroid secretion before implantation in early human pregnancy. J Clin Endocrinol Metab 1993;76:1470-6.

31. Baird DD. Characteristics of fertile menstrual cycles. Scand J Work Environ Health 1999;25 Suppl 1:20-2; discussion 768.

32. Lumley J. Defining the problem: the epidemiology of preterm birth. Bjog 2003;110 Suppl 20:3-7. 


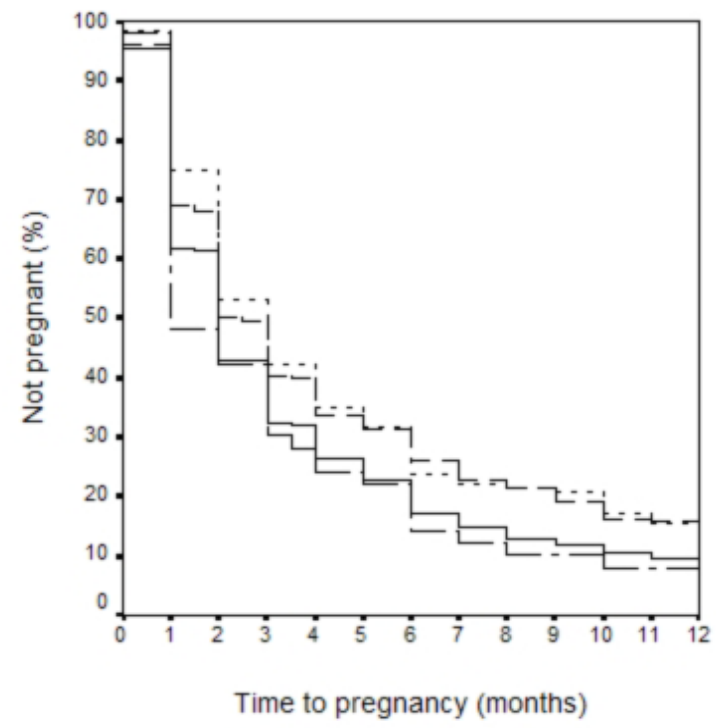

Figure 1. Time to pregnancy for pregnancies ending in single live birth ( $\mathrm{n}=4759$; solid line), early miscarriage ( $n=222$; long dashes), late miscarriage $(n=183$; short dashes), or stillbirth ( $n=52$; long and short dashes) among Swedish women.

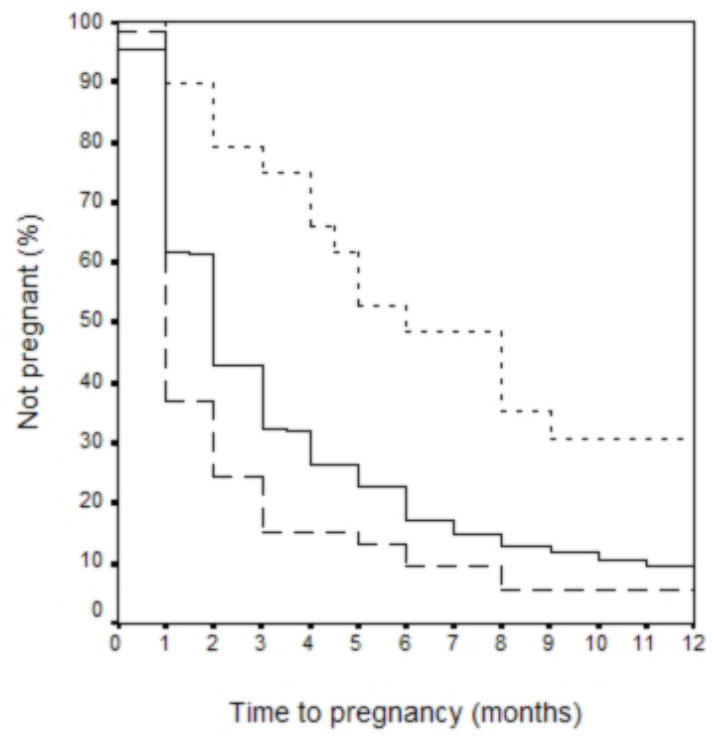

Figure 2. Time to pregnancy for pregnancies ending in single live birth ( $\mathrm{n}=4759$; solid line) or multiple birth ( $\mathrm{n}=57$; long dashes), and extrauterine pregnancies ( $\mathrm{n}=29$; short dashes) among Swedish women. 
Table 1. Women included in a study on a possible association between time to pregnancy (TTP) and pregnancy outcome.

\begin{tabular}{|c|c|c|c|c|c|c|}
\hline Original study & Cohort & $\begin{array}{l}\text { Size of } \\
\text { cohort }\end{array}$ & $\begin{array}{l}\text { Responders to } \\
\text { questionnaire }\end{array}$ & $\begin{array}{l}\text { Response } \\
\text { rate (\%) }\end{array}$ & $\begin{array}{c}\text { Information } \\
\text { available on } \\
\text { TTP }\end{array}$ & $\begin{array}{c}\text { Information } \\
\text { available on } \\
\text { pregnancy }^{\text {outcome }}\end{array}$ \\
\hline \multirow[t]{2}{*}{ Fishermen's wives } & East coast (exposed) & 795 & 506 & 64 & 401 & 378 \\
\hline & West coast (referents) & 1851 & 1090 & 59 & 939 & 870 \\
\hline \multirow[t]{2}{*}{ Fishermen's sisters } & East coast (exposed) & 1241 & 709 & 57 & 563 & 519 \\
\hline & West coast (referents) & 2023 & 1103 & 55 & 866 & 817 \\
\hline \multirow[t]{2}{*}{ Hairdressers } & Hairdressers (exposed) & 5289 & 2626 & 50 & 1595 & 1396 \\
\hline & General population (referents) & 5299 & 2860 & 54 & 1491 & 1322 \\
\hline Total & & 16498 & 8893 & 54 & 5855 & 5302 \\
\hline
\end{tabular}

${ }^{a}$ Excluding induced abortions and still ongoing pregnancies.

Table 2. Women included in a study assessing the possible relation between time to pregnancy and pregnancy outcome.

\begin{tabular}{|c|c|c|c|c|c|c|}
\hline & \multicolumn{2}{|c|}{ Fishermen's wives } & \multicolumn{2}{|c|}{ Fishermen's sisters } & \multicolumn{2}{|c|}{ Hairdressers/referents } \\
\hline & $\mathrm{N}(\%)$ & Median (95\% range) & $\mathrm{N}(\%)$ & Median (95\% range) & $\mathrm{N}(\%)$ & Median (95\% range) \\
\hline Year of pregnancy & & 1976 (1966-1989) & & 1980 (1967-1997) & & 1992 (1984-1998) \\
\hline Age at conception & & $22(17-29)$ & & $24(18-33)$ & & $25(20-32)$ \\
\hline Oral contraceptives & $498(4$ & & $586(4$ & & 1897 & \\
\hline Nulliparous & $1116(\varepsilon$ & & $988(7$ & & 2079 & \\
\hline Non-smoker & $746(60$ & & $753(5$ & & 1571( & \\
\hline
\end{tabular}


Table 3. Pregnancy outcome and time to pregnancy (TTP) among 5302 Swedish women. Fecundability ratios (FRs) with 95\% confidence intervals (CI) are presented for each pregnancy outcome compared to pregnancies ending in the birth of one live child.

\begin{tabular}{|c|c|c|c|c|c|c|c|}
\hline & $\mathrm{N}$ & $\%$ & $\begin{array}{c}\text { Median } \\
\text { (months) }^{\mathrm{a}}\end{array}$ & $\begin{array}{c}\text { All data } \\
\text { FR }(95 \% \text { CI })\end{array}$ & $\begin{array}{l}\text { Excluding first } \\
\text { month conceptions } \\
\text { FR }(95 \% \mathrm{CI})\end{array}$ & $\begin{array}{c}\text { Recall time at most } \\
10 \text { years } \\
\text { FR }(95 \% \mathrm{CI})\end{array}$ & $\begin{array}{c}\text { Recall time at most } \\
5 \text { years } \\
\text { FR }(95 \% \mathrm{CI})\end{array}$ \\
\hline One live child & 4759 & 90 & 2 & 1.00 & 1.00 & 1.00 & 1.00 \\
\hline Multiple birth & 57 & 1 & 1 & $1.62(1.51-2.27)$ & $1.10(0.64-1.89)$ & $1.55(0.96-2.49)$ & $1.35(0.67-2.73)$ \\
\hline Early miscarriage (before week 12) & 222 & 4 & 2 & $0.77(0.66-0.91)$ & $0.79(0.65-0.97)$ & $0.79(0.63-0.98)$ & $0.80(0.60-1.07)$ \\
\hline Late miscarriage (weeks 12-28) & 183 & 3 & 3 & $0.76(0.64-0.91)$ & $0.89(0.72-1.10)$ & $0.84(0.65-1.08)$ & $0.74(0.53-1.05)$ \\
\hline Stillborn child (after week 28) & 52 & 1 & 1 & $1.12(0.80-1.57)$ & $0.85(0.53-1.38)$ & $1.32(0.79-2.20)$ & $0.85(0.36-2.00)$ \\
\hline Extrauterine pregnancy & 29 & 1 & 6 & $0.45(0.28-0.71)$ & $0.57(0.34-0.95)$ & $0.44(0.23-0.87)$ & $0.60(0.26-1.36)$ \\
\hline
\end{tabular}

${ }^{a}$ Excluding women whose TTP is $>2$ months but otherwise unknown.

Table 4. Pregnancy outcome among 5302 Swedish women stratified on the study from which the data originated, and on original cohort affiliation (referent/exposed). Fecundability ratios (FRs) with 95\% confidence intervals (CI) are presented for each pregnancy outcome compared to pregnancies ending in the birth of one live child.

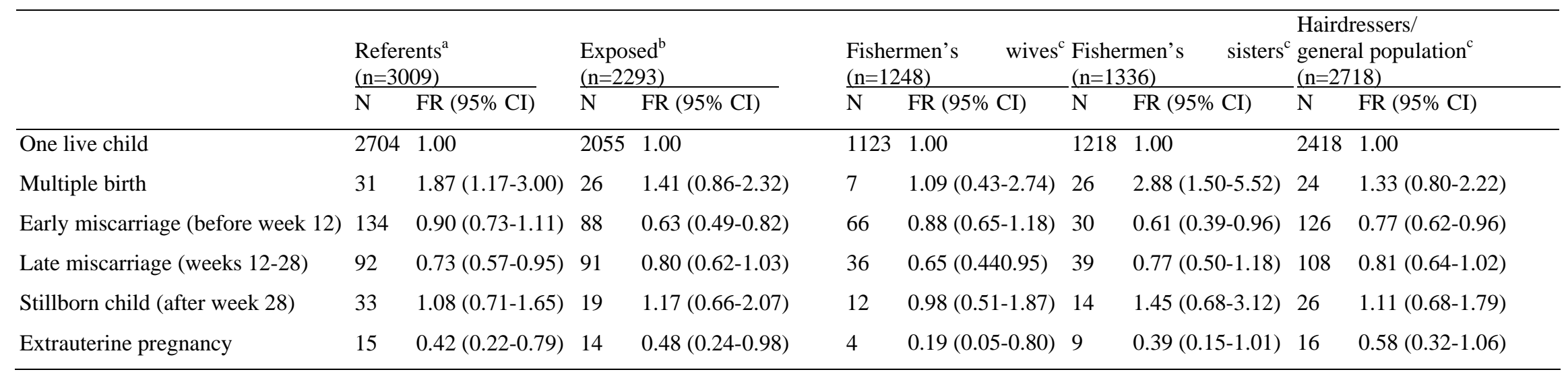

${ }^{a}$ West coast fishermen's wives, west coast fishermen’s sisters, and referents from the hairdresser study

b East coast fishermen's wives, east coast fishermen's sisters, and hairdressers

${ }^{\mathrm{c}}$ Exposed as well as referents 